\title{
High Dynamic Range Content in ISDB-Tb System
}

Diego A. Pajuelo Castro

Paulo E. R. Cardoso

Raphael O. Barbieri

Yuzo lano 


\title{
High Dynamic Range Content in ISDB-Tb System
}

\author{
Diego A. Pajuelo Castro, Paulo E. R. Cardoso, Raphael O. Barbieri, and Yuzo Iano, Senior Member, IEEE
}

\begin{abstract}
Nowadays, the industry is discussing the different requirements for High Dynamic Range television systems. There is no global standard of HDR Content Broadcast yet, which opens countless opportunities for global consumer's electronics in the future. For this reason, we propose an efficient use for Broadcast with backwards compatibility considering to actual standards. Additionally, each processing stage within a broadcast environment is further discussed, considering its limitations. Further objective measures are presented, evaluating different HDR tests.
\end{abstract}

Keywords-Broadcasting; High Dynamic Range; ISDB-Tb; Video System.

\section{INTRODUCTION}

Since the Television birth, the need of transmission of the more realistic scene-referred has always been a challenge in broadcasting service. New technologies and studies have been developed along the last twenty years, as the evolution of the display technology, the advance of broadcast digital television, faster algorithms in the video processing field, and the newest proposals on perceptual coding area. As result, the consumer's electronics demand better user experience and new multimedia content.

High Dynamic Range Content Transmission is the nexthop technology that must be provided to consumer's electronics to reach a better user experience. This technology involves many stages, since capturing, post-processing, coding, and transmission. Each stage may need the development of new standards, digital interfaces, and metadata to ensure the interconnection between different brands. Some industry proposals have been presented along the last years. The BBC and NHK have combined their approaches, the Dolby Labs-Dolby Vision, the Philips, the Samsung, and the Technicolor proposals are well explained in [1], but official global HDR Standard has not been published by the ISO or the ITU.

This paper discusses the possibilities and limitations of Broadcast HDR Content, using the state-of-the art about this subject. Some proposals could be implemented on Broadcast System, reaching better subjective quality and ensure backward compatibility with the actual system.

D. A. P. Castro and R. O. Barbieri are a Master Science Candidates at the State University of Campinas, and researcher at the Laboratory of Visual Communications (e-mail: diegop@decom.fee.unicamp.br).

P. E. R. Cardoso, is $\mathrm{PhD}$ Candidate in Electrical Engineer at the Laboratory of Visual Communications at the State University of Campinas(e-mail: perc@decom.fee.unicamp.br).

Y. Iano is a professor and coordinator of the Laboratory of Visual Communications at the State University of Campinas (e-mail: yuzo@decom.fee.unicamp.br).

The remainder of this paper is organized as follows; Section II presents fundamental concepts of HDR technology, the features of actual broadcast transmission and its limitations. Section III describes the laboratory experiments. Section IV describes the results of objective measurements and Section $\mathrm{V}$ the conclusions of the paper.

\section{FUNDAMENTAL CONCEPTS}

This section describes the fundamental concepts of HDR Technology, as well as the principal topics in terms of brightness and color. First of all, is important to differentiate four important terms that generates confusion. Luminance, " $Y$ ", is a linear-light quantity; proportional to physical intensity weighted by the spectral sensitivity of human vision and is expressed in units of $c d / m^{2}$ ("nits"). Lightness is a non-linear transfer function of luminance that approximates the perception of brightness. This last word, brightness, is the attribute of a visual sensation according to which an area appears to exhibit more or less light, but cannot be measured. Luma Samples has a symbol $Y^{\prime}$, and is an approximation of lightness and indirectly related to the relative luminance, form part of the $Y^{\prime} \mathrm{C} b^{\prime} \mathrm{Cr}$ ' Color Model. [2].

When is referring to Hygh Dynamic Range of human vision, the correct measure is the luminance. Human vision operates between $[100 \mu-10 \mathrm{k}]$ nits and regular TV display operates between [0-100] nits as showed in Fig. 1. According with this idea, the concept of contrast ratio is very important and much discussed topic in HDR displays technology. This factor is the ratio of luminance of the lightest and darkest elements of a scene, or an image, in practical terms, is the brightness difference between white and black. The human perception judged sharper an image reproduced with high simultaneous contrast ratio than another that has higher measured spatial frequency content.

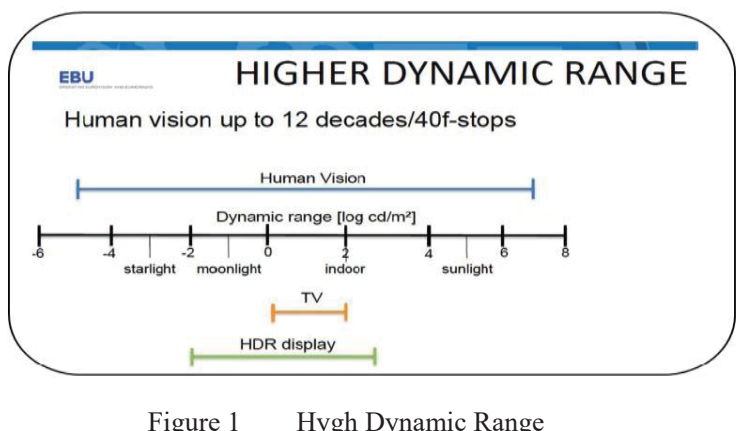

Figure 1 Hygh Dynamic Range 
In accordance with the colorimetry, CIE XYZ tristimulus values form the mathematical coordinates of color space. Each color can be specified by these tristimulus against to its spectrum. CIE 1931 chromaticity diagram represents all the possible colors that a human eye can perceived [2]. Each color is a coordinate in the chromaticity diagram as showed in Fig. 2, actual color space recommendations are just a part of the entire gamut. However, there are enhanced approaches such as the Rec.2020 [3] compared to the Rec.709 [4] in terms of gamut. An intermediate color space is the DCI P3 that is used for digital movie projection from the USAmerican film Industry [5]. The Rec. 709 is commonly used in broadcast transmissions, and form part as MPEG video Standards, such as H.264 [6] and H.265 [7]. HDR technology uses the concept of Wide Color Gamut to represent a color from a scene-referred into a display-referred reliably.

Transfer Function of Tristimulus values responds to physical matter that occurs between the luminance values and human perception. It is demonstrated that luminance is a linear-light quantity and linear-intensity, but has a non-linear response with respect to human perception [2]. Thus, displaying the XYZ linear-values would have a negative subjective perception. Besides, the quantization of this type of material would be inefficient and of worse quality.

Perceptual uniformity is the process of linearize the luminance with respect to human perception; it is when the concept of lightness is created. This idea is based on the property of human contrast sensitivity, which is the ratio of luminance between two adjacent patches of similar luminance [2]. The first correction of luminance values widely adopted is gamma [8], which takes advantage of nonlinearity of the oldest display's CRT.

Perception is the act of extracting meaning from noisy and uncertain sensory signals, many studies have been proposed in the academic community such as [9] that is a human-perception oriented research. One of their advantages is related to the Perceptual domain because is an optimal balance between brightness and coding. For this reason, new standards have proposed new Electrical-Optical Transfer Funcion (EOTF) that works better for actual HDR TV displays and some of them have backward compatibility with regular TV display, as in the case of HLG (Hybrid Log Gamma) [1]. The next proposal is the standard SMPTE ST2084 [10], called PQ-TF. This was developed following the contrast sensitivity function of the human eye as measured by Barten [11]. This system along with metadata can reach backward compatibility in terms of color space, transfer function, white points, and dynamic ranges devices. Another EOTF proposal is done by Philips [1], which follows the Barten curve more smoothly. Phillips proposes its own endto-end transfer characteristic. However it still is not a universal standard or the only one proposal, quite to the contrary, may be necessary a system with end-to-end compatibility. For example an end-to-end Dolby System, with PQ-TF, DCI P3 colour primaries and 5000 nits as peak luminance can be compatible with 100 nits displays and Rec.709 color gamut, or with 1000 nits displays and HLG Transfer Function.

\section{Figure 2 CIE 1931 Chromaticity Diagram}

There are many possibilities, because nowadays there are many sorts of TV displays, the SDR-SDTV such as the CRT Television, the SDR-HDTV, such as LCD or Plasmas, the SDR-UHDTV, such as LED, the HDR-UHDTV, such as

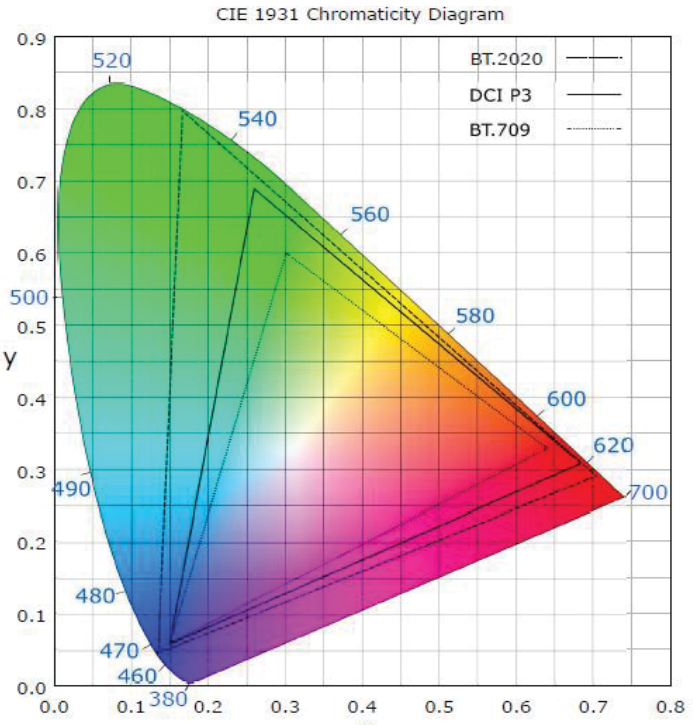

SIM2 [12], for content producers, and OLED for end-users, hence that the future global standard of HDR Content Broadcast Transmission should be manageable and to be designed to the user, ensuring the perceptual rendering intent.

The Broadcast Transmission of digital television is a distinctiveness service that has a large coverage and robustness against the noise. There are four digital television standards mostly used in the world, ISDB-T (Integrated Services Digital Broadcasting or ISDB-Tb, a Brazilian adaptation, ATSC (Advanced Television System Committee), DVB (Digital Video Broadcasting), and DMB (Digital Multimedia Broadcasting). These have been successfully implemented in different countries over recent years. Differences between them are subject to modulation, video codec, and mobility. The last one parameter is implemented with success in ISDB-Tb System, whose main core is the orthogonal frequency domain modulation (OFDM). However broadcast digital television systems are not very flexible respect to scalability, because a Set-top box (STB) is necessary to decode the digital signal and in most cases is not reconfigurable and have limited Profiles and Levels in terms of video codec, bit depth, color model, color space, and digital interface. Due to this, HDR Technology should have the capacity of interact with actual broadcast system and future HDR Systems, as in the past was the color television system with respect to black-and-white television system in the beginnings of broadcast transmission. Each step in the evolution of television always has been thought to have a backward compatibility with old systems.

The actual ISDB-Tb system or the transmission of LDR (Low Dynamic Range) Content has its own features, considering the high definition content. It uses a gamma EOTF, a Rec.709 OETF, sometimes Rec.2020 OETF it is applied, but these are very similar, Rec.709 Color Primaries, and cover display luminance from [0-100] nits. According to the bit depth, it only allows 8 bit encoding, and a H.264 codec with the following constraints: High/Main Profile and Level 4.0. Theoretically, LDR has limited color gamut and even more limited dynamic range.

Consistent with the above explanation, it is clear that is required additional information to allow different conversions, such as color space conversion, tone mapping operation, Transfer function Conversion, color management, white point conversion, and dynamic range devices 
information for interacting between different displays with different nits (luminance).

This paper proposes an efficient Broadcast use for HDR with backwards compatibility considering to actual standards and the limitation of the actual LDR system.

\section{LABORATORY EXPERIMENTS}

For the laboratory experiments, Test Sequences from [13] is used. It is important to note that the capture's process key is to record as much f-stops dynamic range. The resultant data sequences are stored in TIFF files, using the PQ-TF perceptual coding. In Table I, shows the principal details of each Test Sequence and Fig. 4 shows each one of them.

TABLE I. TIFF FILES

\begin{tabular}{|c|c|c|c|c|}
\hline Sequence & Fps & Resolution & $\begin{array}{c}\text { Color } \\
\text { Primaries }\end{array}$ & $\begin{array}{c}\text { Min-Max } \\
\text { luminance } \\
\text { (nits) }\end{array}$ \\
\hline ShowGirl2Teaser & 25 & $1920 \times 1080$ & DCI P3 & $0.005-4000$ \\
\hline FirePlaceTeaser & 24 & $1920 \times 1080$ & DCI P3 & $0.005-4000$ \\
\hline BeerFestTeaser & 25 & $1920 \times 1080$ & DCI P3 & $0.005-4000$ \\
\hline
\end{tabular}

According to the Call For Evidence (CfE) proposed by the ISO group of study [14], the processing of the HDR Test Sequences requires two types of processing to code and decode each sample, the pre and the post processing. The Preprocessing process consists of the following steps:

- Conversion from TIFF to EXR format.

- Apply the PQ-TF on the input video, to work at perceptual domain.

- Conversion of non-linear tristimulus to non-linear color space $Y^{\prime} \mathrm{Cb}{ }^{\prime} \mathrm{Cr}$ '.

- Quantization data from 16 bit floating-point to Bit Depth (8 or 10 bits).

- Finally, Chroma downsampling from 4:4:4 to 4:2:0 is applied on the input video.

The post processing is done in a similar way to the preprocessing, but in reverse. The last process is to apply inverse PQ-TF as shown in Fig. 3. The software 'HDRtools' was used to perform each process [15]. The entire system is shown in Fig. 3.

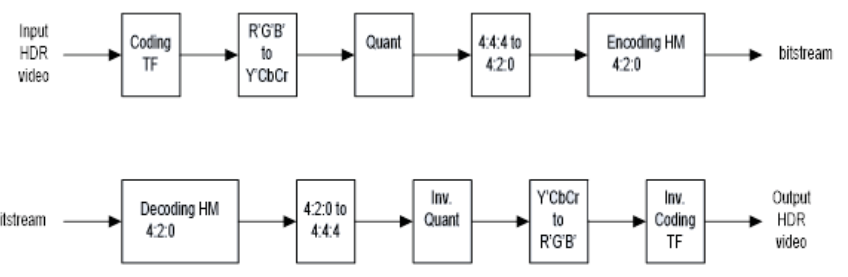

Figure 3 HRD Preprocessing and PostProcessing

The process of quantization from 16 to 8 or 10 bits uses the clipping process to quantize from 8 to 16 bits as well as the inverse process. The conversion from TIFF to EXR format is done because, this format are the 'digital negative' in the HDR environment, because their samples are in linearresponse respect to intensity, this means that it stores the luminance values from the source in comparison to the TIFF Format, who stores the samples after the perceptual encoding, in this case, PQ-TF. Thus it has non-linear samples but a linear-response with respect to human perception.

To backward compatibility with the actual LDR System is important to keep using the Profile and Level allowed in actual ISDB-Tb. The principal parameters are summarized in the Table II. To simulate the stage of encoding 4:2:0, the JM 19.0 H.264/AVC reference [16] software is used.

TABLE II. AVC/H.264 PARAMETERS

\begin{tabular}{|c|c|}
\hline Profile & Main/High \\
\hline Level & 4.0 \\
\hline Chroma Format & $4: 2: 0$ \\
\hline Bit Depth & 8 \\
\hline Resolution & HDTV $(1920 \times 180)$ \\
\hline Coding & Frame \\
\hline NumberBFrames & 2 \\
\hline
\end{tabular}

The goal of the tests is to simulate all the processing stages according to the Fig. 3, taking into consideration the ISDB$\mathrm{Tb}$ constraints, well explained in the fundamental concepts, using different QP (Quantization parameters) for H.264 video codec: $20,22,24,28,32,36$, and 40 and measure objectively difference between Output HDR content respects to the HDR Source. Additional to these results, a 10 bit coding system is used to compare with the 8 bit depth setting.

For a fair comparison, the color primaries used in the laboratory experiments is obtained by the Rec.2020. As it stated above, the Rec.709 is the mostly used at the ISDB-Tb system [17], but it is assumed that it might be a conversion process at the decoder level, which transforms from Rec.709

TABLE III. $\quad 8$ BIT CODING SYSTEM

\begin{tabular}{|c|c|c|c|c|c|c|c|c|c|c|}
\hline $\begin{array}{l}\text { HDR Test } \\
\text { Sequence }\end{array}$ & $\mathbf{Q P}$ & $\begin{array}{c}\text { Bit- } \\
\text { Rate } \\
\text { (Mbps) }\end{array}$ & $\begin{array}{c}\text { Min- } \\
\text { BR } \\
\text { (Mbps } \\
\text { (Mb) }\end{array}$ & $\begin{array}{c}\text { Max- } \\
\text { BR } \\
\text { (Mbps) }\end{array}$ & $\begin{array}{c}\text { m- } \\
\text { PSNR }\end{array}$ & $\begin{array}{c}\text { t- } \\
\text { PSNR } \\
\text { XYZ }\end{array}$ & $\begin{array}{c}\text { tOSNR } \\
\text { XYZ }\end{array}$ & $\begin{array}{c}\text { PSNR } \\
\text { DE100 }\end{array}$ & $\begin{array}{c}\text { PSNR } \\
\text { MD0100 }\end{array}$ & $\begin{array}{l}\text { PSNR } \\
\text { L0100 }\end{array}$ \\
\hline \multirow{4}{*}{ ShowGirl } & 20 & 11,53 & 5,02 & 18,87 & 31,29 & 42,15 & 39,64 & 36,29 & 21,91 & 40,95 \\
\hline & 22 & 7,17 & 3,85 & 11,30 & 30,84 & 41,59 & 39,11 & 36,11 & 21,72 & 40,52 \\
\hline & 24 & 4,63 & 2,91 & 7,05 & 30,44 & 40,95 & 38,49 & 35,84 & 21,52 & 40,05 \\
\hline & 28 & 2,58 & 1,46 & 3,97 & 29,46 & 39,44 & 36,71 & 34,90 & 21,38 & 39,10 \\
\hline
\end{tabular}




\begin{tabular}{ccccccccccc} 
& 32 & 1,53 & 0,80 & 2,39 & 28,34 & 37,72 & 34,99 & 34,15 & 21,22 & 37,97 \\
& 36 & 0,94 & 0,46 & 1,48 & 26,65 & 35,48 & 32,72 & 32,80 & 20,96 & 36,81 \\
& 40 & 0,58 & 0,27 & 0,91 & 25,20 & 33,34 & 30,78 & 31,89 & 20,65 & 35,57 \\
\hline \multirow{5}{*}{ FirePlace } & 20 & 14,59 & 13,19 & 16,62 & 31,10 & 38,66 & 35,37 & 34,73 & 22,22 & 40,15 \\
& 22 & 9,03 & 7,98 & 10,13 & 30,68 & 38,16 & 34,89 & 34,57 & 21,97 & 39,72 \\
& 24 & 5,81 & 4,89 & 6,62 & 30,26 & 37,61 & 34,36 & 34,37 & 21,79 & 39,30 \\
& 28 & 3,07 & 2,45 & 3,68 & 29,44 & 36,49 & 33,26 & 33,89 & 21,46 & 38,44 \\
& 32 & 1,72 & 1,32 & 2,17 & 28,40 & 35,21 & 32,04 & 33,25 & 21,36 & 37,46 \\
& 36 & 1,00 & 0,73 & 1,32 & 27,06 & 33,61 & 30,49 & 32,46 & 21,11 & 36,45 \\
& 40 & 0,57 & 0,40 & 0,79 & 25,72 & 31,93 & 28,90 & 31,77 & 20,74 & 35,35 \\
\hline \multirow{6}{*}{ BeerFest } & 20 & 9,98 & 5,52 & 11,49 & 36,74 & 43,18 & 40,66 & 37,72 & 21,79 & 42,82 \\
& 22 & 7,87 & 4,31 & 9,07 & 36,22 & 42,50 & 40,05 & 37,40 & 21,52 & 42,43 \\
& 24 & 6,02 & 3,29 & 6,97 & 35,50 & 41,61 & 39,24 & 36,95 & 21,19 & 41,94 \\
& 28 & 3,60 & 1,97 & 4,20 & 33,88 & 39,67 & 37,39 & 35,89 & 20,64 & 40,91 \\
& 32 & 2,12 & 1,20 & 2,52 & 32,27 & 37,75 & 35,57 & 34,94 & 20,24 & 39,76 \\
& 36 & 1,26 & 0,74 & 1,53 & 30,22 & 35,57 & 32,25 & 33,66 & 19,85 & 38,55 \\
& 40 & 0,72 & 0,45 & 0,91 & 28,61 & 33,67 & 31,38 & 32,82 & 19,51 & 37,29 \\
\hline \hline
\end{tabular}

TABLE IV. $\quad 10$ BIT CODING SYSTEM

\begin{tabular}{lcccccccccc}
\hline \hline $\begin{array}{c}\text { HDR Test } \\
\text { Sequence }\end{array}$ & QP & $\begin{array}{c}\text { Bit-Rate } \\
\text { (Mbps) }\end{array}$ & $\begin{array}{c}\text { Min- } \\
\text { BR } \\
\text { (Mbps) }\end{array}$ & $\begin{array}{c}\text { Max- } \\
\text { BR } \\
\text { (Mbps) }\end{array}$ & $\begin{array}{c}\text { m- } \\
\text { PSNR }\end{array}$ & $\begin{array}{c}\text { t- } \\
\text { PSNR } \\
\text { XYZ }\end{array}$ & $\begin{array}{c}\text { tOSNR } \\
\text { XYZ }\end{array}$ & $\begin{array}{c}\text { PSNR } \\
\text { DE100 }\end{array}$ & $\begin{array}{c}\text { PSNR } \\
\text { MD0100 }\end{array}$ & $\begin{array}{c}\text { PSNR } \\
\text { LO100 }\end{array}$ \\
\hline \multirow{5}{*}{ ShowGirl } & 20 & 10,63 & 4,53 & 17,58 & 31,56 & 42,41 & 39,60 & 36,55 & 22,06 & 41,16 \\
& 22 & 6,71 & 3,55 & 10,68 & 31,04 & 41,73 & 38,81 & 36,24 & 21,85 & 40,68 \\
& 24 & 4,40 & 2,73 & 6,74 & 30,55 & 41,01 & 38,09 & 35,88 & 21,69 & 40,17 \\
& 28 & 2,50 & 1,38 & 3,83 & 29,56 & 39,54 & 36,68 & 35,05 & 21,49 & 39,17 \\
& 32 & 1,50 & 0,78 & 2,34 & 28,43 & 37,77 & 34,99 & 34,21 & 21,37 & 38,02 \\
& 36 & 0,93 & 0,46 & 1,47 & 26,98 & 35,78 & 33,12 & 33,21 & 21,13 & 36,88 \\
FirePlace & 40 & 0,57 & 0,27 & 0,90 & 25,49 & 33,57 & 31,04 & 32,37 & 20,78 & 35,60 \\
\hline & 20 & 13,48 & 12,06 & 15,45 & 31,25 & 38,82 & 35,57 & 34,87 & 22,35 & 40,26 \\
& 22 & 8,50 & 7,40 & 9,61 & 30,83 & 38,29 & 35,05 & 34,69 & 22,14 & 39,80 \\
& 24 & 5,54 & 4,65 & 6,35 & 30,43 & 37,75 & 34,52 & 34,48 & 21,87 & 39,36 \\
& 28 & 2,99 & 2,38 & 3,58 & 29,58 & 36,60 & 33,40 & 33,98 & 21,56 & 38,47 \\
& 36 & 1,70 & 1,30 & 2,13 & 28,59 & 35,34 & 32,19 & 33,38 & 21,44 & 37,49 \\
& 1,00 & 0,73 & 1,31 & 27,33 & 33,80 & 30,73 & 32,68 & 21,19 & 36,49 \\
& 40 & 0,56 & 0,40 & 0,78 & 26,04 & 32,12 & 29,17 & 32,05 & 20,78 & 35,37 \\
\hline \multirow{5}{*}{ BeerFest } & 20 & 9,34 & 5,01 & 10,82 & 37,08 & 43,50 & 41,10 & 37,96 & 21,80 & 43,05 \\
& 22 & 7,42 & 3,97 & 8,61 & 36,47 & 42,75 & 40,40 & 37,55 & 21,52 & 42,60 \\
& 24 & 5,72 & 3,07 & 6,64 & 35,72 & 41,80 & 39,53 & 37,07 & 21,20 & 42,05 \\
& 28 & 3,47 & 1,89 & 4,07 & 34,08 & 39,80 & 37,64 & 35,98 & 20,65 & 40,97 \\
& 32 & 2,08 & 1,16 & 2,48 & 32,44 & 37,86 & 35,77 & 34,99 & 20,26 & 39,81 \\
& 36 & 1,25 & 0,73 & 1,53 & 30,68 & 35,84 & 33,73 & 33,97 & 19,87 & 38,65 \\
& 40 & 0,72 & 0,45 & 0,90 & 29,03 & 33,90 & 31,81 & 33,16 & 19,53 & 37,33 \\
\hline \hline
\end{tabular}

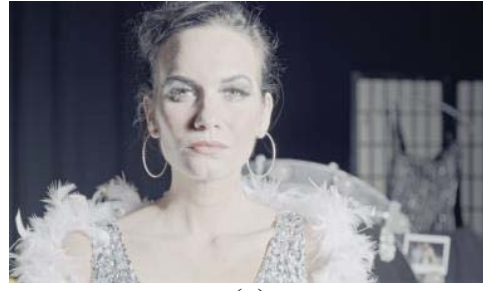

(a)

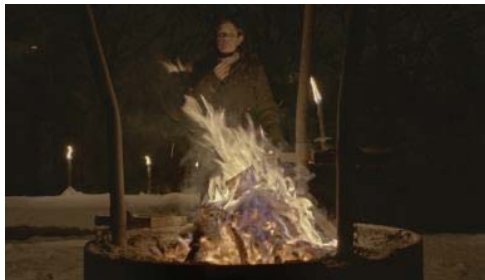

(b)

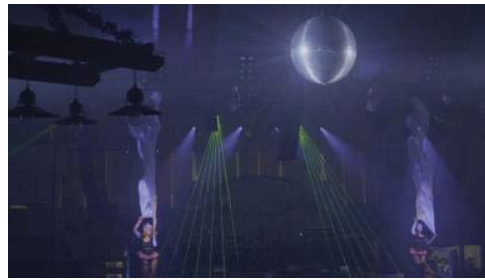

(c)

Figure 4 HDR Test Sequences, (a) ShowGirl; (b) FirePlace; (c) BeerFest 
Color Primaries to Rec.2020 Color Primaries, ensuring the perceptual rendering and artistic intent. The objective measures have been successfully used in [18] and the software 'HDRTools' is also used to get the HDR Metrics.

\section{RESULTS}

Objective results for an 8-bit coding system, are showed in Table III for the three type of sequences, ShowGirl, FirePlace and BeerFest for different QP parameters. These values are acceptable according to [18], and have no noticeable distortions. It is clear when the QP value increases its value, the objective values (measured in $\mathrm{dB}$ ) are lower and it is possible to evaluate the content as poorer quality, however the bit-rate could be optimal for situations when bandwidth is limited. It is important to note that encoding with 10 bits generates less bit-rate than 8 bits [19]. The difference between the best m-PSNR value and the worst m- PSNR value is $6.09 \mathrm{~dB}$ for ShowGirl, $5.38 \mathrm{~dB}$ for FirePlace, and $8.73 \mathrm{~dB}$ for BeerFest because they have different brightness and artistic intent.

When is tested the same process above, but with a 10 bit coding system, using the same test sequences, the objective results are showed in Table IV, the same behavior is repeated when QP increases as explained above, but the reason of this additional test is to measure if there is a great difference respect to a 8 coding system. Hence, according to the results, the difference oscillates between 0.2 and $0.3 \mathrm{~dB}$. The main reason is well explained in [20], the minimal bits requires for no visible banding or artifacts depends on the source content, using the Just Noticeable Difference (JND) theory [21]. In this case, the 3 types of sequences have no difficult images and are adequate for a ISDB-Tb System.

An 8-bit system is still useful to send HDR Content with backward compatibility; however the use of extrainformation could be a compatible option for darker images, better than moving to 10-bit system. Also, may be unaffordable and technically unfeasible in the short term.

\section{CONCLUSION}

In this paper, we have presented a proposal to adapt the High Dynamic Range Content with backward compatibility in the ISDB-Tb System. For this, we use the perceptual coding called PQ-TF [10]. As part of the validations, we evaluate the whole processing objectively, comparing the Output HDR content respects to the HDR Source in an 8-bit system with different QP. For higher QPs, the objectives results are acceptable and demonstrate a reliable system, reaching the possibility of taking advantage of the actual system. Additional comparison with a 10-bit system, demonstrates that for adequate source content, the objective difference are minimal and does not justify moving to that system.

Besides it is important to mention that the HDR full-user experience will depend on OOTF (Opto-optical Transfer Function), which has the function of applying the rendering intent of the content producer.

\section{FUTURE WORKS}

The development of a new universal standard to carry the information of transform function, peak luminance, white point or tone mapping operators to interact between distinct sort of displays, inside the SEI (Supplemental Enhanced Information) messages. Furthermore, test the HLG (HybridLog Gamma) with the ISDB-Tb System for backward compatibility purposes and compare different EOTF's subjectively in different displays.

\section{ACKNOWLEDGMENT}

The authors would like to thanks CAPES, CNPq, and Fapesp; also the other teachers and staff of DECOM, FEEC, and UNICAMP.

\section{REFERENCES}

[1] Society of Motion Picture and Television Engineers, SMPTE, "Study Group Report High Dynamic-Range (HDR) Imaging Ecosystem" , 2015.

[2] C. Poynton, "Digital Video and HDTV: Algorithms and Interfaces", Morgan Kaufman Publishers, 2003.

[3] ITU-R BT.2020, "Parameter Values for ultra-high definition television systems for production and International programme exchange," International Telecommunication Union, Dec. 2015.

[4] ITU-R BT.709, "Parameter values for the HDTV standards for production and international programme exchange," International Telecommunication Union, May. 2015.

[5] SMPTE RP 431-2, "Digital Cinema Quality", 2011.

[6] Advanced Video Coding for Generic Audiovisual services, document ITU-T Rec.H.264 and ISO/IEC 14496-10 (AVC) (Version 22), Feb. 2012 .

[7] High Efficiency Video Coding, document ITU-T Rec. H.265 and ISO/IEC 23008-2 (Version 2), Oct. 2014.

[8] ITU-R BT.1886, "Reference electro-optical transfer function for flat panel displays used in HDTV studio production", International Telecommunication Union, March. 2011

[9] C. R. Sims, "Rate-distortion theory and human perception," Cognition, vol. 152, pp. 181-198, 2016

[10] SMPTE ST 2084, "High Dynamic Range Electro-Optical Transfer Function of Mastering Reference Display", 2014.

[11] P. G. J. Barten, "Contrast Sensitivity of the Human Eye and Its Effects on Image Quality," 1999, SPIE.

[12] HDR47ES6MB | SIM2 High Dynamic Range Visualization. [online] Available at: http://hdr.sim2.it/hdrproducts/hdr47es6mb [Accessed 12 Jul. 2016].

[13] J. Froehlich, S. Grandinetti, B. Eberhardt, S. Walter, A. Schilling and H. Brendel, "Creating cinematic wide gamut HDR-video for the evaluation of tone mapping operators and HDR-displays", IS\&T/SPIE Electronic Imaging, 2014

[14] A. Luthra, E. Francois, and W. Husak, "Call for Evidence (CfE) for HDR and WCG Video Coding," in ISO/IEC JTC1/SC29/WG11 MPEG2014/N15083. Geneva, Switzerland: Feb. 2015

[15] A.M. Tourapis and D. Singer, "HDRTools: Software updates," ISO/IEC JTC1/SC29/WG11, Doc. M35471, Geneva, Switzcerland, Feb. 2015.

[16] JM Reference Software, Version 19.0. [Online]. Available: http://iphome.hhi.de/suehring/tml/, accessed July 12, 2016.

[17] Technical Specifications for Brazilian Digital terrestrial television, http://www.abnt.org.br/ (ABNT 15604 Brazilian Technical norm for digital TV receivers)

[18] P. Hanhart, M. Rerabek, T. Ebrahimi, "Subjective and Objective Evaluation of HDR Video Coding Technologies", $8^{\text {th }}$ International Conference on Quality of Multimedia, 2016

[19] D. Ruiz, S. Sladojevic, D. Culibrk, "Comparison of compression performance of 10-bit vs. 8-bit depth, under H.264 Hi422 profile", $11^{\text {th }}$ International Conference on Telecommunication in Modern Satellite, Cable and Broadcasting Services, IEEE, 2013, no. 1, pp. 119-122

[20] S. Miller, M. Nezamabadi, S. Daly, "Perceptual Signal Coding for More Efficient Usage of Bit Codes", SMPTE Motion Im. J., no. 4, pp. 52-59, 2013

[21] M. K. Stern and J. H. Johnson, "Just noticeable difference," in Corsini Encyclopedia of Psychology. Hoboken, NJ, USA: Wiley, 2010

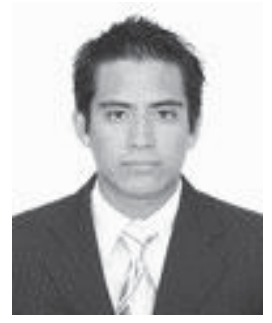

Diego A. Pajuelo Graduate in Electrical Engineering from the Peruvian University of Applied Sciences (UPC), Lima, Peru in 2012. He is currently working towards his Master degree in Sciences and Telecommunications at the State University of Campinas (UNICAMP). His research Interests 
are Video and audio coding, Image processing, Digital television and Satellite communications.

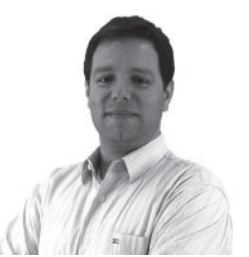

Paulo E. R. Cardoso Holds a degree in Electrical Engineering from the FEECUNICAMP (2002) and a MSc degree in Electrical Engineering (Electronics) by DEMIC-FEEC-UNICAMP (2005). He is currently a $\mathrm{PhD}$ candidate in the LCV-DECOM-FEEC-UNICAMP, searching Digital TV. Licensed from the post of Specialist in Regulating the National Telecommunications Agency Anatel, where it operates in coordination of grants and resources to the provision, working with the licensing and amendment of technical characteristics of broadcasting stations. Previously, he served in the surveillance in broadcasters and in the solution of problems of radio interference in any system of telecommunication. He was responsible for the Technical Regulation to Broadcasting in Modulated Frequency and analysis of processes of technical feasibility for inclusion or amendment of the Basic Plan of Distribution Channels of Broadcasting in Modulated Frequency. He participated as an observer in the Federal Government in testing of Digital Radio Broadcasting, both in tests of American Standard - HD Radio, in 2008 and 2012, as in tests of the European standard - DRM, in 2010. He has worked as a telecommunications researcher of the Foundation Center for Research and Development in Telecommunications - CPqD.

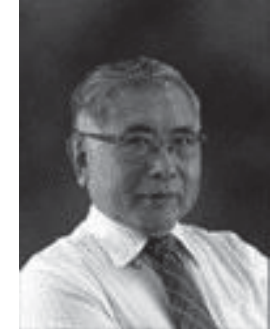

Yuzo Iano Prof. Yuzo Iano is the head and founder of the Laboratory of Visual Communications since 1972. He obtained his BSc (1972), MSc (1974) and $\mathrm{PhD}$ (1986) in Electrical Engineering at University of Campinas, SP-Brazil. Research Interests: Digital Signal Processing (images/audio/video), Digital TV, 4G (LTE) and 5G Cellular Networks, Pattern Recognition, Smart Cities, Smart Grid, Internet of Things.

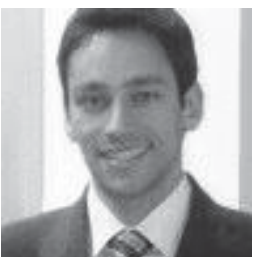

Raphael O. Barbieri Graduated in Computer Engineering from FEECUNICAMP (2007) and MBA in Project Management from IBE-FGV (2014). Member of Technical Module of SBTVD Forum and Product Manager at EiTV. He is currently working on his Master's degree in Electrical Engineering at FEECUnicamp.

Research Interests: Digital TV, ISDB-Tb and Broadcast Engineering. 